\title{
Sick and solo: a qualitative study on the life experiences of people living with HIV in Somalia
}

This article was published in the following Dove Medical Press journal: HIVIAIDS - Research and Palliative Care

\author{
Abdulwahab M Salad' \\ Abdinasir Mohamed ${ }^{2}$ \\ Omar B Da'ar ${ }^{3}$ \\ Abdirizak Abdikarim ${ }^{4}$ \\ Prabhjot Kour ${ }^{5}$ \\ Mary Shrestha ${ }^{6}$ \\ Abdi A Gele 6 \\ 'School of Public Health and \\ Research, Somali National University, \\ Mogadishu, Somalia; ${ }^{2}$ The Department \\ of Public Health, Ministry of Health, \\ Puntland State, Somalia; ${ }^{3}$ St. Mary's \\ University of Minnesota, Winona, \\ MN, USA; ${ }^{4} \mathrm{HIV}$ Program, Ministry \\ of Health, Mogadishu, Somalia; \\ ${ }^{5}$ Norwegian National Advisory Unit \\ on Concurrent Substance Abuse and \\ Mental Health Disorders, Innlandet \\ Hospital Trust, Ottestad, Norway; \\ ${ }^{6}$ Migrants Health Unit, Norwegian \\ Institute of Public Health, Oslo, \\ Norway
}

Background: HIV stigma and the resultant fear of being identified as HIV-positive can compromise the effectiveness of HIV programs by undermining early diagnosis and antiretroviral treatment initiation and adherence of people living with HIV (PLHIV). In the wake of the longstanding conflict in the country, little is known about the life experiences of PLHIV in Somalia. Methods: A qualitative study using unstructured interviews was conducted in Somalia from September to December 2017. A convenience sampling approach was used to recruit 13 participants, including 10 persons who live with HIV and three senior officials who work for the HIV program at the Ministry of Health. Data were analyzed using a thematic analysis.

Results: Our findings show that PLHIV are alienated and prefer to isolate themselves due to widespread stigma subjected to them by their family members, society, employers, and health providers, which continue to undermine the scale-up of testing and treatment of PLHIV in Somalia. Consequently, they are reluctant to seek voluntary diagnosis and treatment of HIV. They often come to know about their status when their partners are found HIV positive, they are tested for other clinical purposes, or when an individual's health deteriorates, and all other means fail to work in improving his/her situation. The study also pointed out a shortage of facilities that provide HIV diagnosis, counseling and treatment in Somalia.

Conclusion: Addressing stigma and discrimination subjected to PLHIV are critical to a successful HIV response in Somalia. To successfully address stigma, HIV programs need evidence on effective interventions at individual, community, and societal levels in order to strategically incorporate stigma and discrimination reduction into national HIV programs.

Keywords: HIV stigma, PLHIV, discrimination, Somalia

\section{Introduction}

HIV infection and AIDS have emerged as a global public health concern with a death toll of about one million people in $2016,{ }^{1}$ and an infection rate of 5,000 people per day. ${ }^{2}$ An estimated 36.7 millions of people in the world are living with HIV, and although about half of the people living with HIV (PLHIV) are receiving antiretroviral therapy (ART), the prevalence and its death rate are still significant. About 1.3 million PLHIV lived in conflict and emergency settings in Sub-Saharan Africa in 2013, where they often lack access to essential HIV services and suffer as a result of shortages that could have been avoided. ${ }^{3}$ The United Nation AIDS (UNAIDS) stated that "unless HIV is addressed in conflict settings such as Somalia, we will not reach zero new HIV infections, zero discrimination and zero AIDS-related deaths". ${ }^{3}$

Somalia is an East African country and little is known about HIV situation in the country though estimates show a prevalence of $1 \% .{ }^{4}$ Sentinel surveillance data in 2010
Norwegian Institute of Public Health, Besøksadresse: Sandakerveien 24 C, Bygg DII, Postboks 4404 Nydalen, 0403 Oslo, Norway

Tel +479682 2030

Email Abdi.gele@fhi.no 
showed a prevalence of $1.2 \%$ among pregnant women aged 15-24 years, $12.3 \%$ among patients with sexually transmitted diseases, and 5.6\% among TB patients in Somaliland regions. ${ }^{5}$ Although the prevalence is low, due to the ongoing conflict, HIV sentinel surveillance cannot reach most of the affected areas, and the current HIV infection problem may be greater than the figures indicate. ${ }^{6}$ However, it is believed that the actual prevalence might be higher because of the unwillingness of PLHIV to reveal their status because of stigma and the political insecurity, making it difficult to conduct reliable nationwide research. ${ }^{4,7}$ This is supported by earlier data analysis which indicated that HIV infection rates were up to $18 \%$ among people with TB and 5.2\% among female sex workers accompanied with poor utilization of condoms by both men and women. ${ }^{8}$ There are also indications of a high mortality rate of HIV-infected people. Out of 23,500 people who were infected with HIV in Somalia, only $11 \%$ were under ART in $2016 .^{9}$

Coverage of HIV services in Somalia is low, ${ }^{10}$ with only 16 centers that provide ART in the country in 2017. A study carried out in Mogadishu stated that a widespread HIV stigma limits the disclosure of one's HIV status to families and communities, which creates a risk of self-isolation and ill-health. ${ }^{11}$ Stigma has been defined as "an attribute that is deeply discrediting, and discrimination directed at people perceived to have AIDS or HIV, and the individuals, groups and communities with which they are associated". ${ }^{12}$ PLHIV in some communities in Africa avoid seeking treatment at clinics for fear of having their status disclosed or of suffering further stigma and discrimination based on their HIV status. ${ }^{13} \mathrm{HIV}$ stigma persists as a barrier to a concerted action to reduce the transmission of HIV, as it impedes prevention and treatment efforts. ${ }^{14}$ This may entice a person to hide his/ her serostatus, to self-isolate and refrain from seeking treatment, further, which may escalate the possibility of engaging in unprotected sex. ${ }^{15}$ The aim of this study is to explore the experiences of PLHIV to generate new insights and inform researchers, program managers, and policymakers on what problems PLHIV are facing, in order to improve the national response to HIV and to scale up testing and treatment of HIV in Somalia.

\section{Methods}

A qualitative study using unstructured interviews was conducted in Somalia from September to December 2017. A snowball sampling of 13 participants, aged $\geq 25$ years, was conducted in Somalia. Participants were recruited through contact with the Network of PLHIV, which is an organization that unites PLHIV in Somalia and aims to see PLHIV have access to treatment and care of HIV and to be able to use these services free of discrimination and stigma. With some exceptions, most of the network members are strictly secretive. The first author, who is a researcher at Somali National University together with an assistant, conducted the interviews. Researchers contacted the leader of the network who linked the research team with the members of the network upon an explanation of the study. The leader, in turn, contacted members of the network and explained about the study, emphasizing the need for their voluntary participation and to recruit other members to participate in the study. Those who were recruited and who accepted to participate were called by the research team and were explained about the objectives of the study and the freedom to participate. The participants were then asked about the best way for them to be interviewed and a venue of their choice. All participants agreed to be interviewed via telephone. Moreover, three service providers, who work for the National HIV Program, were purposely recruited. The research team also explained to the providers about the study before obtaining verbal consent.

We followed common research ethics principles in carrying out this study, including informed consent, where their rights to refuse, withdrawal, and confidentiality were explained to participants. Subsequently, verbal consent was obtained from each participant. Verbal informed consent was acceptable and approved by Ethical Board, and this study was conducted in accordance with the Declaration of Helsinki. The Somali National University's Ethical Board approved the execution of the study with approval no: EB-0148.

Prior to conducting the interviews, the research team developed trust and good rapport with the participants. In addition, participants were informed of their rights to withdraw from the study at any time, and assurance of the confidentiality of their information was given. The interviews were conducted in Somali, the native language of the participants and interviewers.

\section{The content of the interview}

The participants were asked about their reaction to their HIV diagnosis, how they felt when they sought treatment and disclosed their HIV status to their close family, friends, and networks. In addition, their health situation during preand post-initiation of treatment was sought. We explored their access to treatment, care, and health information, in addition to their experiences of stigma and discrimination. 
Furthermore, the factors that prevent people from utilizing existing preventive and treatment services were explored. Finally, the acceptable means of delivering preventive health information and services were explored through participants' perspectives. The interview process continued until saturation was achieved.

\section{Analysis}

The second, sixth, and the last authors transcribed the interviews verbatim. The transcripts were thoroughly read (more than three times) by the second author and the last author. We used Nvivo 12 Plus for the analyses. Thematic analysis was used to identify and analyze important themes, ${ }^{16}$ with the coding process involving recognition of and encoding the identified themes prior to interpretation. Themes that emerged from the interviewees' narratives were pieced together to form a comprehensive picture of the participants' shared experiences. ${ }^{17}$ The themes were divided into categories based on the participants' experiences of living with HIV.

\section{Results}

This study included persons who live with HIV (PLHIV) and health service providers. Out of the ten PLHIV who were interviewed, eight were females and two were males. Additionally, the research team interviewed three senior officials, who work at the HIV program, Ministry of Health. The participants' lives were affected by HIV and they lived with the scourge from 2 to 16 years. The participants were residing in Mogadishu, Marko, Shalanbood, and Kismayo cities of Somalia. Three major themes were identified that answered the aim of the study (Table 1). The themes were as follows: responses to test results, facets of stigma, and shortage of HIV services.

Table I Themes and categories that emerged

\begin{tabular}{|l|l|}
\hline Themes & Categories \\
\hline $\begin{array}{l}\text { Response to } \\
\text { test result }\end{array}$ & $\begin{array}{l}\text { What makes them seek HIV testing } \\
\text { Reaction to the positive test } \\
\text { Counseling } \\
\text { Perceived source of infection } \\
\text { Optimism about own health }\end{array}$ \\
\hline Facets of stigma & $\begin{array}{l}\text { Stigmatization and rejection within the family and } \\
\text { society } \\
\text { Stigmatization at the health facilities } \\
\text { Stigmatization at work places } \\
\text { Fear of HIV disclosure to avoid stigma } \\
\text { Reducing stigma through patients' perspectives }\end{array}$ \\
\hline $\begin{array}{l}\text { Shortage of HIV } \\
\text { services }\end{array}$ & Shortage of health services \\
\hline
\end{tabular}

\section{Theme I: responses to test results}

Participants expressed their reactions to the diagnosis of HIV. This theme was divided into the following four categories, which was categorized as per the participants' responses: what pushed them to seek HIV testing, reactions to positive tests, counseling, and the alleged source of infection.

\section{What pushed them to seek HIV testing}

The majority of the study participants reported that they were not aware of their HIV status until they become severely sick and hospitalized, whereas a few participants mentioned that they were tested because their spouses were diagnosed as HIV positive. Participants' responses are as follows:

First, I had TB and went to the doctor and tested for TB. I was diagnosed as TB positive and I started taking TB drugs, but my situation did not change while all other people who were taking TB drugs in the hospital were recovering. Then the doctor asked for general blood examination, and I accepted. When the doctor took the blood and examined, they found that I have HIV. (Participant 2)

I was tested in Marko hospital, and before that, I used to go to pharmacies and MCHs. When I reached to Marko hospital, I was very ill and very weak. (Participant 3)

I became sick and had diarrhoea for six days, and then I was taken to Marko hospital. First, they gave me TB drugs, but my health was not getting better, so they suspected that I have HIV. They conducted blood check-up and the result became HIV positive. Then I was transferred to a Mercy hospital. (Participant 9)

Some of the study participants stated that they were called for a blood test after their spouses were found HIV positive.

After marriage, I went to Uganda for treatment of bullet injury. After I came back, I was getting sick, and the wife was already very ill and died. Her sister told me that my ex had HIV and died as result of AIDS. After that, I contacted the hospital, where, after blood test, I was confirmed HIV positive. (Participant 10)

My husband became HIV positive, so they asked him if he has a wife. Then I went for blood test and I was confirmed HIV positive. (Participant 6)

The service providers reported that people who come for HIV tests have mostly already developed AIDS. Service providers also indicated that people rarely come for voluntary testing, making an early diagnosis of HIV infection a rare endeavor in Somalia. 
Most Somalis do not go for voluntary testing. We find they are positive with HIV when they are in a critical condition. They are often transferred from another hospital or they are diagnosed when they are donating blood, sometimes during delivery among pregnant women. It is rare to find someone who wants test voluntarily or come to our voluntary testing centers. Most people we see are in the late-stage and have developed AIDS. (Service Provider 1)

\section{Reactions to positive test}

Majority of the participants had negative reactions to HIVpositive results. They are shocked and/or in denial mode to accept the test result while some even become aggressive toward the health care providers. Below are some quotes from the participants:

When I was tested positive for the first time, I could not believe it and I didn't accept that I have the disease. I was in shock, so I gave a wrong number to the doctors so that they cannot find me. I was hiding for almost three months. (Participant 7)

I was in shock, felt hopeless, and I was near to be getting mad. I was discriminated and isolated.

My ex-wife was diagnosed as HIV positive, so she told me to go the hospital and get a test for HIV. We went together to Mercy hospital. I was tested, and the result was positive for HIV. I did not accept it, and went to Dr Duffle hospital for retesting, the result was the same, I was confirmed positive for HIV. I accepted it and started taking the drugs. (Participant 8)

\section{Counseling}

Most of the participants stated that they had pre- and post-test counseling. They were counseled to accept their new status, to undergo the ART and to disclose their HIV status to at least their close family members. Here are some of their quotes:

I travelled to Mogadishu, and I was welcomed by HIV staff and one of the staff told that even he himself is positive for HIV, so I should calm down. He gave me counseling. (Participant 2)

The service providers, who were interviewed also, confirmed that people are provided counseling before and after the test for PLHIV. However, both participants and providers agreed that people come to testing facility at a late stage of the disease and that is when counseling is provided.

When performing blood test, we tell them that test includes

HIV test and we demonstrate them of how test works. We also counsel them about the results, and help to disclose their status. (Service Provider 3)

However, as one of the participants reported, counseling is not provided to everyone. A woman stated that she did not receive counseling, and health providers never informed her about her HIV-positive status.

After a while, I questioned my dad why I am taking this drug for a long time. My dad told me that I am HIV positive and told me to calm down. (Patient 1)

\section{Perceived source of infection}

Several participants reported that they were infected with HIV by their spouses. Female participants blamed their husbands for the transmission of the infection. While one male participant stated that he became infected after having sex with women in his early adulthood, another male participant also related the source of infection to his wife, who died of AIDS. Because sexually transmitted HIV is associated with promiscuity and adultery, people should attribute their infection to an acceptable source.

I asked my dad why am I positive? Used to hear that people who commit adultery, get infected with HIV and I am married women and have not had adultery or infidelity. Then my dad told me maybe your husband was positive and transmitted it to you, later I divorced him. (Participant 1)

I think I have got the infection through sexual contact. I was young, and I did not know about this disease and never heard of it and did not know about condoms or sexually transmitted diseases. So, that is how I got it. (Participant 7)

One of the service providers also reported that PLHIV, mainly women, link the HIV infection to their husbands. Some of them associate the source of the infection to surgery or injection.

Most of the patients say that they got infected through medical operations and injection, very few people may tell you that they got infected through sexual intercourse and they link to their partners mostly their husbands. (Service Provider 2)

\section{Optimism about own health}

Participants indicated optimism about their health after taking ART, which encouraged them to adhere to treatment and can be a lesson to others to come forward to accept their HIV status and seek treatment. 
Before I was infected, I was healthy and had normal body weight, but when I became HIV positive I became sick. I started feeling better after I started ART. (Participant 4)

Thanks to God I am feeling better, I have received ART from Mercy hospital. I am in a state of recovery now. (Participant 8)

\section{Theme 2: facets of stigma}

The study participants reported that they were stigmatized, ashamed, discriminated, rejected, and isolated by their community, family, friends, the general society as well as employers and health providers. Participants stated that their community holds stigmatizing attitude and exhibits stigmatizing behaviors toward PLHIV. Some participants have lost their jobs because of discrimination, some participants were abandoned by their husbands or wives, while others were forced not to disclose their HIV status for fear of discrimination, while others avoided diagnostic test or hid their status or even default from the ART just to avoid rejection and discrimination.

\section{Stigmatization and rejection by the family and society}

Participants stated that they were made to feel ashamed and were insulted because of their HIV status. They reported daily experiences of stigmatization, verbal abuse, and rumors about their HIV status. All the participants reported that they felt isolated, rejected, and that they lost contact with their families, friends, neighbors, and the community.

My mother rejected and left me alone. She did not talk to me until she died. (Participant 4)

I do not interact with neighbours; they know about my situation. They do not speak to me (Participant 1).

My family and people around me treated me badly. I was very sick and had a baby, I could not wash clothes because I had an infection and scar in my hand, no one helped me, even I had nothing to eat but no one wanted to visit to me (Participant 3).

When I was confirmed positive with HIV ...my uncle said to other family member "don't bring her home, let her stay in the hospital for the rest of her life". Other members of my family and friends discriminated me and didn't want me to be one of them, the only person who is on my side is my dad (Participant 1).

When I became positive, my husband divorced me because of HIV (Participant 2).

\section{Stigma at work place}

PLHIV were fired from their jobs and discriminated by their colleagues. They reported being called derogatory names and subjected to inhumane conditions. The statements of the PLHIV also included accounts of mistreatment and being likened to animals such as dogs.

I was working as welder. Now aim not working because my work colleagues discriminated me and they didn't want me work with them, just because of I am an HIV positive. (Participant 8)

One day I went to a restaurant, when I sat on the table there was another guy who was sitting there, when he saw me he moved to another table because he knew that I am HIV positive. When I went to a ceremony, people were eating food together, but I wasn't allowed to share with them because of my HIV positive status. Even sometimes in the mosque some people will not stand and pray close to me. I need to be patience otherwise I will be mentally ill when I face such discrimination. (Participant 7)

They told me that I and the dogs should share same plate and eat together, I and the dogs will be in the same hospital. (Participant 2)

\section{Stigma at the health facility}

PLHIV are not only stigmatized and rejected by their families and society but also discriminated by the health care professionals. Some of them shared their experiences of witnessing a forced discharge from the health care facilities. In one instance, an expectant mother was denied a C-section procedure after she was found to be HIV positive. Their quotes are as follows:

Although we have very low antenatal care coverage, every woman who goes to $\mathrm{MCH}$ or a hospital during her pregnancy undergo HIV test. Pregnant women who are HIV positive give birth in specific hospitals, like our hospital. Recently, we have experienced a case of HIV positive woman who attended a hospital for delivery. She was in a delivery suite when they found that she is HIV positive, they discharged her immediately and rejected to deliver in their hospital. (Service Provider 1)

Pregnant women who live with HIV are not treated well in other hospitals. When they find them HIV positive, they immediately send them to our Hospital (Banadir hospital). Even sometimes in the middle of operations, they discharge the patient with tools and equipment they were using for the operation left on her side. (Service Provider 2) 


\section{Fear of HIV disclosure to avoid stigma}

All participants stated about their feelings of fear and rejection in relation to the disclosure of their HIV status. Participants who have disclosed and those who have not disclosed their HIV-positive status reported that revealing and sharing their HIV status with other people, including close family and friends, will put them in trouble and stigmatization. The main reason for not wanting to tell and share their HIV status is the fear of stigma and discrimination.

They discriminate you if they get to know about your HIV positive status. I did not tell my family and friends that I am HIV positive. I am scared if I disclose this, I will be discriminated. (Participant 5)

People do not know that I have HIV, because if I tell them, they will discriminate me. (Participant 5)

There are people who have signs and symptoms of HIV, but don't want to go for diagnosis because they are afraid of stigma and rejection. One of my friends, who I suspected that he had HIV, refused to go for blood test, and he died in few months later. The reason of his refusal was that he was afraid of rejection and stigma. (Participant 8)

Health providers approved participants' narratives regarding people's fear of disclosure of their HIV status. According to the health providers, stigma associated with the HIV is detrimental to people's motivation in seeking HIV diagnosis and treatment.

When someone is suspected to have HIV, rumors spread in the town, and for curiosity, people in the neighborhood begin to scrutinize the movement of the person such as his/ her visit to the hospital. For that reason, PLHIV may not come to the hospital for diagnosis and treatment because if neighbors see them around the hospital, they will label and stigmatize them. (Provider 1)

I myself have about 10 patients who do not want to come to the HIV care center to collect their treatment. I deliver the treatment to them at universities, schools, or any other place of their choice. If I do not deliver the treatment to them, they may never come to the HIV center to collect their treatment. Coming to HIV treatment centers is extremely stigmatizing. (Provider 1)

One of the participants even reported that he defaulted the treatment because he is overwhelmed by the stigma and discrimination subjected to him on a daily basis.

Stopped taking the drugs, and my condition begin to worsen. When I was taking drugs regularly, I was getting better, but after I was stigmatized and bullied, I started hating the drug and stopped taking it. (Participant 1)

\section{Road to reduce stigma through participants' perspectives}

The participants presented their perspectives about addressing and reducing stigma and improving the life of PLHIV. They were of the opinion that raising awareness and educating the people about HIV is important to reduce the stigma. Participants also pointed out the importance of moral support to improve the lives of PLHIV and to ensure their rights for dignity and respect. Some of the participants mentioned that supportive behavior from people around and praying to God would also reduce stigma.

By increasing awareness and health education among the public will reduce the stigma. (Participant 8)

I think the lives of PLHIV can be improved if they receive moral support. (Participant 10)

We provide counselling to people who come to us, but we do not go to the town for awareness creation. General population have no awareness about HIV. There are organizations that conduct awareness-workshops, which often last for one-day. That is not enough. There is a need for aggressive media based awareness campaign involving the health providers, policy makers, religious leaders, and community leaders. (Provider 2)

\section{Theme 3: shortage of comprehensive HIV services}

All participants reported that HIV services for PLHIV are extremely scarce. Most participants stated that the availability of ART providing centers are very limited, and the long distance and expensive fares to such facilities limit the PLHIV in accessing prompt treatment and counseling services.

When I became positive, the doctor told me to go to Mogadishu and asked me to arrange my flight. I told him that I do not have enough money to fly to Mogadishu. After that, he spoke to the charity which helped me to finance the trip to Mogadishu. (Participant 2)

I have to travel to get the medications from Marko hospital every two months; I cannot get it from Shalanbood where I live. (Participant 3)

We have several centers that take HIV test but we have only two confirmatory test centers in Mogadishu (Banadir and Mercy). These two centers are the only two that provide ART drugs to the PLHIV. In whole of Somalia, we have 
only nine centers that provide ART to PLHIV. (Service Provider 1)

\section{Discussion}

This study explored the situations and experiences of PLHIV in Somalia. The result shows that these participants did not seek voluntary HIV diagnosis and treatment, but they come to know about their status when their partners are found HIV positive, they are tested for other clinical purposes, or when an individual's health deteriorates, and all other means fail to work in improving his/her situation. Prompt testing, diagnosis, and treatment before developing clinical AIDS and subsequent opportunistic diseases can prevent illness and improve survival and reduce transmission..$^{18}$ Delay in diagnosis adversely affects both disease prognosis at the patient level and transmission at the community level. ${ }^{19}$ People receiving HIV diagnosis late in the course of infection are often, severely immunocompromised, more likely to present with co-morbidities such as tuberculosis, and have short-term mortality. Our study shows that people often present to health facilities with clinical AIDS manifestations. This is accompanied by the fact that Somalis have stigma toward HIV. That is why there are many valid cultural reasons why HIVpositive individuals in Somalia do not disclose their status and seek early testing and care, including fear of familial or partner abandonment, rejection, stigma, and discrimination, which may eventually block the demand for diagnosis and treatment. ${ }^{20}$ To respond effectively to the epidemic, general health education and high-level activism through the media should be conducted in order to raise awareness about the HIV among the public, so that PLHIV are treated with dignity, and each person in Somalia feel safe in seeking early diagnosis, counseling, and treatment of the infection.

The study pointed out a number of entrenched sociocultural and structural issues that act as barriers to HIV prevention in Somalia. Of particular concern is stigma subjected to PLHIV by their family members, society, employers, and health providers, which continue to undermine the scale-up of testing and treatment of PLHIV in Somalia. Our findings are in line with a previous study reporting that PLHIV face discrimination and isolation and are often denied access to their rights and basic services. ${ }^{21}$ Globally, stigma and discrimination are recognized as facilitators in the spread of HIV infections, barriers in the practice of safe and effective HIVprevention behaviors, and significant obstacles in the access of HIV care, treatment, and support services. ${ }^{22-24}$ Stigma and subsequent fear of being identified as having HIV or AIDS may discourage people from being tested, from accessing medical services and medications, and from disclosing their HIV status to others. ${ }^{25}$ Researchers described stigma experienced by PLHIV as a manifestation in the tensions that exist between the cultural norms and attitudes about HIV and the implications of these cultural narratives for PLHIV. ${ }^{25}$ This process begins as a person confronts two domains of a stigma that arise from cultural beliefs about HIV: blame and stereotypes and fear of transmitting the disease through all types of contacts. In this context, individuals are seen as "contaminated" and linked to the stereotypes of promiscuity and that may be the reason why some of our study participants sought health care after they have already developed clinical AIDS. According to the executive director of UNAIDS, "whenever AIDS has won, stigma, shame, distrust, discrimination and apathy was on its side. Every time AIDS has been defeated, it has been because of trust, openness, dialogue between individuals and communities, family support, human solidarity, and the human perseverance to find new paths and solutions". The general population in Somalia including health service providers were reported to hold stigmatizing attitudes to PLHIV and exhibit stigmatizing behaviors toward them; this could lead to an undesirable consequence given the fact that HIV flourishes in areas where stigma and discrimination against PLHIV are common. ${ }^{26}$ According to study respondents, HIV-related stigma and discrimination block the provision of services, such as employment and the demand for services within health care settings. In Africa, HIV-related stigma has been associated with reduced uptake of voluntary counseling and testing ${ }^{26}$ and increased sexual risk-taking behavior in the general population. ${ }^{23} \mathrm{~A}$ study conducted with Somali participants in Sweden revealed that $\mathrm{HIV}$ is perceived as a shame and disgraceful act linked to adultery and fornication. ${ }^{27}$ This revelation is consistent with our findings, where participants presented fear of being labeled and ashamed as sinners and consequently delayed seeking HIV diagnosis and treatment. However, unlike the previous study in Mogadishu, ${ }^{11}$ our study reports a worrying discrimination of the PLHIV and denial of services by health providers, consistent with earlier findings in other sub-Saharan African countries. ${ }^{6}$ The fear and insufficient knowledge of HIV infection among health providers, as well as lack of awareness of stigma and its harmful consequences on social judgments, prejudices, and stereotypes against PLHIV may hamper the efforts to control HIV infection in Somalia. Addressing stigma and discrimination subjected to PLHIV is critical to a successful HIV response in Somalia.

The shortage of facilities that provide HIV diagnosis, counseling, and treatment is another critical challenge in HIV 
control program in Somalia. Private health care system is the dominant health providing facilities in Somalia, ${ }^{28}$ and our findings show that private providers are reluctant to provide services to PLHIV, which questions the overall efficiency of national HIV programs in Somalia. A private hospital owner in Mogadishu in an interview with Aljazeera TV stated, "We are a private hospital and if those patients not suffering from HIV or AIDS found out we treat people with AIDS or HIV in our hospital, they will all discharge themselves and we won't have any patients and we won't have any business after that. To treat HIV positive or AIDS patients will be economic suicide". In line with this report, our study shows that private hospitals discriminate PLHIV from health services for fear of losing customers. Lack of access to private facilities for HIV care means that the PLHIV must rely on HIV services that are provided by few public hospitals in major towns in the country. This delayed access to care may result in the clinical presentation of more advanced stages of the HIV disease, which is, in fact, the case in Somalia according to our finding. A timely access to care has been shown to substantially reduce morbidity and mortality, reduce HIV transmission risk, and improve the overall quality of life of PLHIV. ${ }^{19,23}$ The ART is freely provided by the NGOs in Somalia, but its availability is confined to few hospitals, and many PLHIV are still not able to afford to pay indirect costs, such as traveling and accommodation cost. There is an evidence that people who experience access problems such as the high cost for transportation, long distance to facilities, and stigma do not seek HIV care. ${ }^{29}$ People seeking diagnosis and treatment of HIV in Somalia may visit multiple health providers prior to reaching the one that provides ART, which is costly for the patient in terms of money and time. Consequently, patients who are poor lack resources for transportation, compelling them not to seek health care and potentially transmitting the disease.

The study has limitations. First, the reported result is the perspective of 13 participants, but it is unclear whether this is the view of the majority of PLHIV in Somalia. Moreover, the providers' experience with stigma and discrimination was not explored during the interviews. Despite the limitations, this study identified the pervasive stigma and discrimination subjected to PLHIV in Somalia.

\section{Conclusion and recommendations}

According to the findings of this study, social disgrace related to living with HIV is pervasive in Somalia. The stigma and discrimination are among the foremost barriers to HIV prevention, treatment, care, and support, and thus, it undermines the HIV prevention efforts by making people afraid to seek HIV information, services, and modalities to reduce their risk of infection and to adopt safer behaviors. Thus, study participants presented their perspectives in engaging in stigma reduction strategy to improve the lives of PLHIV. The strategies forwarded by participants included health education to communities, public awareness on HIV, and moral support to PLHIV. Aggressive awareness campaigns about HIV across the country are critical to destigmatize HIV. Somalia's powerful media network should be used for awareness campaigns against stigma subjected to PLHIV, as study participants, particularly health providers, see the media as one of the critical enablers in HIV stigma reduction. Nonetheless, HIV programs in Somalia need evidence on effective interventions at individual, community, and societal levels in order to strategically incorporate stigma and discrimination reduction into national HIV programs. Therefore, an intervention study aimed at reducing stigma and discrimination targeted at different sectors, including families, communities, and institutions (eg, health care settings and the workplace) with the engagement of multiple target audiences and potential change agents, is recommended. Moreover, there needs to be an emphasis on inspiring private health providers to provide dignified services to PLHIV at elevated levels to end the epidemic. Finally, it is critical to promote a rights-based approach to HIV testing and counseling services; this would help normalize them in health facilities and in communities. Of vital importance is to improve people's perceptions about the benefits of prompt HIV testing and counseling, which may impart a direct impact on the uptake of services in addition to the early diagnosis and initiation of ART.

\section{Data sharing statement}

The data used to support the findings of this study may be released upon application to the Somali National University Ethical Committee. It can be contacted through the first author, Abdulwahab M Salad: abdisalad@snu.edu.so.

\section{Acknowledgments}

The authors acknowledge the help they received from the ARV clinics staff and Network Organization for HIV/AIDS patients, Somalia, in conducting this study. This work was supported by the School of Public Health and Research, Somali National University. The abstract of this paper was presented at the 2nd International Conference on Sexually Transmitted Diseases, Infections and AIDS as an abstract presentation with interim findings. The abstract was published at www.pulsus.com, and it can be accessed at: https://std.cmesociety.com/2018/abstract/sick-and-solo-a-qualitative-studyon-lived-experience-of-living-with-hiv-aids-in-somalia. 


\section{Disclosure}

The authors report no conflicts of interest in this work.

\section{References}

1. World Health Organization [homepage on the Internet]. HIV/AIDS Online Q \& A; 2017. Available from: http://www.who.int/features/ qa/71/en/. Accessed January 31, 2018.

2. The United Nations AIDS (UNAIDS) [homepage on the Internet]. Country Factsheets Somalia 2016. HIV and AIDS Estimates; 2017 Available from: http://www.unaids.org/en/regionscountries/countries/ somalia/. Accessed November 1, 2018.

3. UNAIDS. HIV in emergency contexts. 2015. Available from: http://www. unaids.org/sites/default/files/media_asset/20150729_UNAIDS_PCB36_ ppt_15-13_HIV_emergency_contexts.pdf. Accessed February 20, 2019.

4. The United Nations AIDS (UNAIDS) [homepage on the Internet]. The Joint United Nations Programme on HIV/AIDS. Mogadishu-HIV in a Time of Unrest; 2012. Available from: http://www.unaids.org/en/ resources/presscentre/featurestories/2012/july/20120726mogadishu. Accessed January 31, 2018.

5. Abdalla E, Ekanem E, Said D, Arube P, Gboun M, Mohammed F. The need for a comprehensive response to HIV/AIDS in north-western Somalia: evidence from a seroprevalence survey. East Mediterr Health J. 2010;16(2):141-145.

6. Ahmed BH, Giovagnoli MR, Mahad H, Tarsitani GG. Burden of HIV/ AIDS infection before and during the Civil war in Somalia. East Mediterr Health J. 2010;16(8):907-909.

7. Kulane A, Owuor J, Sematimba D, Abdulahi S, Yusuf H, Mohamed L. Access to HIV care and resilience in a long-term conflict setting: a qualitative assessment of the experiences of living with diagnosed HIV in Mogadishu, Somali. Int J Environ Res Public Health. 2017; 14(7):721.

8. Kriitmaa K, Testa A, Osman M, et al. HIV prevalence and characteristics of sex work among female sex workers in Hargeisa, Somaliland, Somalia. AIDS. 2010;24 Suppl 2:S61-S67.

9. The United Nations Development Programme (UNDP); 2016. Responding to HIV and AIDS in Somalia - Annual Report 2015. Available from: http://www.so.undp.org/content/dam/somalia/Reports/Q4-2015/ UNDP\%20HIV\%202015\%20Annual\%20Report\%20-\%20Final.pdf. Accessed February 20, 2019.

10. Eskild A, Helgadottir LB, Jerve F, Qvigstad E, Stray-Pedersen S, Løset A. [Induced abortion among women with foreign cultural background in Oslo]. Tidsskr Nor Laegeforen. 2002;122(14):1355-1357.

11. Kulane A, Owuor J, Sematimba D, Abdulahi S, Yusuf H, Mohamed L. Access to HIV care and resilience in a long-term conflict setting: a qualitative assessment of the experiences of living with diagnosed HIV in Mogadishu, Somali. Int J Environ Res Public Health. 2017; 14(7):721.

12. Goffman E. Stigma: Notes on the Management of Spoiled Identity. New Jersey: Prentice-Hall, Simon and Schuster; 1963.
13. Campbell C, Foulis CA, Maimane S, Sibiya Z. "I have an evil child at my house": stigma and HIV/AIDS management in a South African community. Am J Public Health. 2005;95(5):808-815.

14. Judgeo N, Moalusi KP. My secret: the social meaning of HIV/AIDS stigma. SAHARA J. 2014;11(1):76-83.

15. The Joint United Nations Programme on HIV/AIDS (UNAIDS) [homepage on the Internet]. Mogadishu-HIV in a Time of Unrest; 2012. Available from: http://www.unaids.org/en/resources/presscentre/ featurestories/2012/july/20120726mogadishu. Accessed June 22, 2016.

16. Braun V, Clarke V. Using thematic analysis in Psychology. Qual Res Psychol. 2006;3(2):77-101.

17. Taylor S, Bogdan R. Introduction to Qualitative Research Methods: The Search for Meanings. New York, NY: Wiley and Sons; 1984.

18. Trepka MJ, Fennie KP, Sheehan DM, Lutfi K, Maddox L, Lieb S. Late HIV diagnosis: differences by Rural/Urban residence, Florida, 2007-2011. AIDS Patient Care STDS. 2014;28(4):188-197.

19. Carrizosa CM, Blumberg EJ, Hovell MF, et al. Determinants and prevalence of late HIV testing in Tijuana, Mexico. AIDS Patient Care STDS. 2010;24(5):333-340.

20. Lazarus JV, Himedan HM, Østergaard LR, Liljestrand J. HIV/AIDS knowledge and condom use among Somali and Sudanese immigrants in Denmark. Scand J Public Health. 2006;34(1):92-99.

21. Hadjipateras A. Unravelling the Dynamics of HIV/AIDS-Related Stigma and Discrimination: The Role of Community-Based Research: Case Studies of Northern Uganda and Burundi. London, Kampala, Nairobi; Agency for Co-operation and Research Development (ACORD); 2004.

22. Brown L, Macintyre K, Trujillo L. Interventions to reduce HIV/AIDS stigma: what have we learned? AIDS Educ Prev. 2003;15(1):49-69.

23. Kalichman SC, Simbayi LC. HIV testing attitudes, AIDS stigma, and voluntary HIV counselling and testing in a black township in Cape Town, South Africa. Sex Transm Infect. 2003;79(6):442-447.

24. Mahajan AP, Sayles JN, Patel VA, et al. Stigma in the HIV/AIDS epidemic: a review of the literature and recommendations for the way forward. AIDS. 2008;22(Suppl 2):S57-S65.

25. Sayles JN, Ryan GW, Silver JS, Sarkisian CA, Cunningham WE. Experiences of social stigma and implications for healthcare among a diverse population of HIV positive adults. J Urban Health. 2007;84(6): 814-828.

26. United Nations Programme on HIV/AIDS. Reduction of HIV-related stigma and discrimination. UNAIDS 2014 Guidance Note; 2014.

27. Aden AS, Dahlgren L, Guerra R. Experiences against HIV/ AIDS/STDS of Somalis in exile in Gothenburg, Sweden. Ann Ig 2004;16(1-2):141-155.

28. Gele AA, Ahmed MY, Kour P, Moallim SA, Salad AM, Kumar B. Beneficiaries of conflict: a qualitative study of people's trust in the private health care system in Mogadishu, Somalia. Risk Manag Healthc Policy. 2017;10:127-135.

29. Yehia BR, Stewart L, Momplaisir F, et al. Barriers and facilitators to patient retention in HIV care. BMC Infect Dis. 2015;15(1):246.
HIV/AIDS - Research and Palliative Care

\section{Publish your work in this journal}

HIV/AIDS - Research and Palliative Care is an international, peerreviewed open access journal focusing on advances in research in HIV its clinical progression and management options including antiviral treatment, palliative care and public healthcare policies to control viral spread. The journal is included in PubMed. The manuscript man-

\section{Dovepress}

agement system is completely online and includes a very quick and fair peer-review system, which is all easy to use. Visit http://www.dovepress. $\mathrm{com} /$ testimonials.php to read real quotes from published authors. 